\title{
负载型贵金属催化剂用于室温催化氧化甲醛和 室内空气净化
}

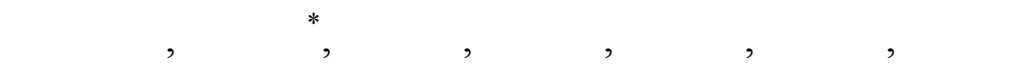

(1) 中国科学院生态环境研究中心, 北京 100085;

(2) 北京亚都空气污染治理技术有限责任公司, 北京 102206;

(3) 北京新奥集团有限公司, 北京 100029

* 联系人, E-mail: honghe@rcees.ac.cn

2008-10-10 收稿, 2008-11-22 接受

国家科技支撑计划绿色奥运科技专项(编号：2007BAK12B12)、国家自然科学基金(批准号：20607029)、国家高技术研究发展计划(编号: 2007AA061402)和中国科学院优秀博士学位论文获得者科研启动专项资金资助项目

摘要 随着室内空气污染状况受到日益关注, 许多研究者开始致力于开发高效、安全、经济的 室内空气净化材料和技术. 本文介绍了我们研究室内甲醛净化催化剂的过程, 比较了室温下负 载型贵金属催化剂利用空气中的氧气完全氧化甲醛的性能, 阐述了 $\mathrm{Pt} / \mathrm{TiO}_{2}$ 催化剂的高效活性 本质, 明确了贵金属催化剂上催化氧化甲醛的反应机理. 应用研究方面, 建立了成型催化剂和 甲醛净化组件的制备生产工艺, 研发了新型空气净化器和通风管道内应用的净化机. 上述两种 产品实现了高效、安全和经济的室内甲醛净化, 已经供应市场并应用于 2008 年北京奥运会部 分室内空气质量的保障, 为绿色奥运、科技奥运、人文奥运做出了应有的贡献, 并将惠及千家 万户百姓的生活.

关键词 室内空气 甲醛 室温催化氧化 贵金属 反应机理 空气净化 北京奥运
随着人们物质文化生活水平的提高, 室内装修 已成为时尚, 随之而来的便是室内空气污染越来越 严重. 甲醛是来自家具和装修材料中最严重的污染 物, 它是一种具有较高毒性的物质, 在我国有毒化学 品优先控制名单上高居第二位, 并且已经被世界卫 生组织确定为致癌和致畸形物质 ${ }^{[1 ~ 3]}$. 长期接触低剂 量甲醛可引起慢性呼吸道疾病, 鼻咽癌、细胞核的基 因突变、白血病等疾病. 近几年来, 随着各方面的宣 传和人们科普知识的增加, 公众对室内装修引发的 一系列污染问题越来越重视, 因此解决室内环境污 染成为研究者所关注的热点问题之一.

传统用于室内甲醛净化的方法主要有吸附技术、 光催化技术、低温等离子体技术以及催化氧化技术 ${ }^{[4]}$. 吸附技术主要采用活性炭和分子篮等高比表面材料 来吸附甲醛, 以达到净化空气的目的, 但由于吸附材 料吸附能力有限, 需定期再生或更换, 易产生二次污
染. 光催化技术主要利用纳米 $\mathrm{TiO}_{2}$ 作为光催化剂, 通 过活性氢氧自由基和超氧自由基的作用, 在室温条 件下就可以分解甲醛. 该技术对于净化室内空气具 有良好的应用前景, 但目前光催化反应还主要需要 紫外激发光源, 对可见光利用效率低, 另外还存在催 化剂失活的问题. 常压下空气放电产生的低温等离 子体中包含电子、离子、氧活性物种和激发态分子等 有极高化学活性的物种, 可以打开污染气体分子的 化学键, 使很多高活化能的化学反应得以发生, 从而 达到处理室内空气中有机污染物的目的. 但是该技 术在对空气放电过程中容易产生氮氧化物及臭氧等 二次污染物, 所以目前在空气净化器内应用较少.

热催化氧化技术在较低的温度下就可以将甲醛 完全分解为无害的 $\mathrm{H}_{2} \mathrm{O}$ 和 $\mathrm{CO}_{2}$, 因而成为最有应用前 景的净化甲醛的方法. 关于甲醛的催化氧化已有一 定的研究报道. 早在 1986 年, Saleh和Hussian ${ }^{[5]}$ 就发 
现甲醛在干净 $\mathrm{Ni}, \mathrm{Pd}$ 和Al的氧化薄膜上分解时有 $\mathrm{CO}_{2}$ 生成, 当温度高于 $423 \mathrm{~K}$ 时甲醛会在氧化膜上完全分 解. Sekine等人 ${ }^{[6]}$ 使用活性炭颗粒和氧化锰研制出一 种板状空气净化材料来去除甲醛, 实验发现通过这 种材料甲醛在室温下就可以分解为 $\mathrm{H}_{2} \mathrm{O}$ 和 $\mathrm{CO}_{2}$, 但是 经过一段时间后该材料的甲醛去除效率会大大降低, 显示室温下氧化锰表现为甲醛的氧化剂而非催化剂. Álvarez-Galván等人 ${ }^{[7]}$ 考察了负载型 $\mathrm{Mn} / \mathrm{Al}_{2} \mathrm{O}_{3}$ 和 $\mathrm{Mn}-\mathrm{Pd} / \mathrm{Al}_{2} \mathrm{O}_{3}$ 催化剂对甲醛和甲醇的氧化活性, 发现 当反应温度高于 $363 \mathrm{~K}$ 时甲醛在 $\mathrm{Mn}-\mathrm{Pd} / \mathrm{Al}_{2} \mathrm{O}_{3}$ 催化剂 上可以完全转化为 $\mathrm{CO}_{2}$ 和 $\mathrm{H}_{2} \mathrm{O}$. Tang等人 ${ }^{[8,9]}$ 研制了 $\mathrm{MnO}_{x}-\mathrm{CeO}_{2}$ 及 $\mathrm{Ag} / \mathrm{MnO}_{x}-\mathrm{CeO}_{2}$ 复合金属催化剂用于催 化分解甲醛, 发现在 $100^{\circ} \mathrm{C}$ 时可将 $500 \mathrm{ppm}$ 的甲醛完 全分解. 最近, $\mathrm{Li}$ 等人 ${ }^{[10]}$ 研究了 $\mathrm{FeO}_{x}$ 负载 $\mathrm{Au}$ 催化剂催 化氧化甲醛的性能, 发现 $7.1 \% \mathrm{Au} / \mathrm{FeO}_{x}$ 催化剂可使 甲醛在 $80^{\circ} \mathrm{C}$ 时实现完全转化. 尽管研究者在催化氧 化甲醛方面做了许多工作, 但采用上述催化剂, 催化 氧化甲醛的操作温度通常远高于室温, 难以满足室 内空气净化所需的常温常压、能耗低的要求, 因此只 有开发出室温、常压下能完全氧化甲醛为 $\mathrm{H}_{2} \mathrm{O}$ 和 $\mathrm{CO}_{2}$ 的材料, 才有希望实现催化氧化技术在室内空气甲 醛净化方面的实际应用.

基于上述原因, 我们致力于研发在室温条件下 能够完全催化氧化甲醛的催化剂. 本文对我们在室 内甲醛净化催化剂开发方面的研究成果进行了综述, 在简要回顾初期研究思路以及 $\mathrm{Cu} / \mathrm{Al}_{2} \mathrm{O}_{3}$ 催化剂净化 甲醛研究的基础上, 主要介绍了具有室温催化氧化 甲醛性能的 $\mathrm{Pt} / \mathrm{TiO}_{2}$ 催化剂的开发历程, 阐述了 $\mathrm{Pt} / \mathrm{TiO}_{2}$ 催化剂的高效活性本质和该催化剂常温催化 氧化甲醛的反应机理 ${ }^{[11 ~ 13]}$, 同时对该催化剂在实际 应用研究方面的成果进行了总结. 目前, 我们已经开 发了以 $\mathrm{Pt} / \mathrm{TiO}_{2}$ 催化材料为核心的新型空气净化器和 通风管道内应用的净化机, 空气净化器已经上市销 售，通风管道内应用的净化机也已经在奥运工程上 得到应用. 可以预见, 该催化材料在净化室内甲醛方 面具有良好的应用前景.

\section{1 室温高效催化净化甲醛催化剂研究}

我们有关室内甲醛室温催化净化的研究工作是 从 2004 年开始的. 2003 年我国“非典”爆发, 2004 年初 我们承担了国家科技攻关项目“新型环保、安全、经
济的室内空气消毒、净化方面研究”, 主要研究室温 下室内空气的催化氧化消毒, 也有部分催化净化室 内空气化学污染的内容. 室温下催化杀菌研究的成 功 ${ }^{[14,15]}$, 大大增强了我们利用空气中的氧气室温催 化氧化甲醛的信心. 现代表面科学的研究结果显示, 室温下氧气吸附在许多过渡金属和一些贵金属表面 时, 可以解离成吸附状态的氧原子或带电荷的过氧 或超氧自由基, 所以从原理上讲, 完全有可能利用空 气中丰富的氧, 通过催化活化实现甲醛的室温完全 氧化.

$\mathrm{Cu} / \mathrm{Al}_{2} \mathrm{O}_{3}$ 催化剂是一种良好的多功能氧化催化 剂，常被用于 $\mathrm{CO}$ 、甲烷、甲醇、乙醇以及乙醛的完 全氧化 ${ }^{[16-20]}$. 所以, 在研究初期我们首先对 $\mathrm{Cu} / \mathrm{Al}_{2} \mathrm{O}_{3}$ 催化剂催化氧化甲醛的性能进行了详细的研究. 结果 表明 ${ }^{[21]}$, 在室温空气的条件下, $\mathrm{Cu} / \mathrm{Al}_{2} \mathrm{O}_{3}$ 不具备催化 氧化甲醛为 $\mathrm{CO}_{2}$ 和 $\mathrm{H}_{2} \mathrm{O}$ 的活性, 其去除甲醛的能力主 要来自 $\mathrm{Al}_{2} \mathrm{O}_{3}$ 对甲醛的吸附作用. 吸附在 $\mathrm{Cu} / \mathrm{Al}_{2} \mathrm{O}_{3}$ 上 的甲醛会被部分氧化为表面甲酸盐物种; 当吸附达 到饱和后, $\mathrm{Cu} / \mathrm{Al}_{2} \mathrm{O}_{3}$ 完全被甲酸盐物种所覆盖而失去 去除甲醛的能力; 负载 $\mathrm{Cu}$ 组分对载体 $\mathrm{Al}_{2} \mathrm{O}_{3}$ 的去除甲 醛能力并没有明显的影响, 但却能大大降低吸附物 种在载体 $\mathrm{Al}_{2} \mathrm{O}_{3}$ 上的分解温度; 通过升温脱附处理, 表面甲酸盐物种将完全分解为无害的 $\mathrm{CO}_{2}$ 和 $\mathrm{H}_{2} \mathrm{O}$, 并 从催化剂表面脱附出来, 同时 $\mathrm{Cu} / \mathrm{Al}_{2} \mathrm{O}_{3}$ 获得再生而 可以重新利用. 显然, 尽管 $\mathrm{Cu} / \mathrm{Al}_{2} \mathrm{O}_{3}$ 具有较强的去除 甲醛的能力, 但其并没有室温催化完全氧化甲醛为 $\mathrm{CO}_{2}$ 和 $\mathrm{H}_{2} \mathrm{O}$ 的活性.

贵金属以其优良的催化氧化活性常作为催化氧 化有机物的催化剂，已广泛应用于很多工业领域，所 以我们设想通过贵金属活性组分与载体的匹配来设 计氧化催化剂, 以期达到无需特定光源而室温条件 催化完全氧化甲醛的目的, 并最终篮选出了能常温 催化分解甲醛的 $\mathrm{Pt} / \mathrm{TiO}_{2}$ 催化剂. 图 1 给出了 $\mathrm{TiO}_{2}$ 负 载的 4 种贵金属 $(\mathrm{Pt}, \mathrm{Rh}, \mathrm{Pd}, \mathrm{Au})$ 催化剂和纯 $\mathrm{TiO}_{2}$ 载体 对甲醛的催化氧化活性随温度的变化趋势. 可以看 出 5 种催化剂对甲醛的氧化活性由高到低的排列顺 序为 $\mathrm{Pt} / \mathrm{TiO}_{2}>>\mathrm{Rh} / \mathrm{TiO}_{2}>\mathrm{Pd} / \mathrm{TiO}_{2}>\mathrm{Au} / \mathrm{TiO}_{2}>>\mathrm{TiO}_{2}$. $\mathrm{Pt} / \mathrm{TiO}_{2}$ 展示了极佳的催化氧化甲醛活性, 在 50000 $\mathrm{h}^{-1}$, 室温条件下, 就能完全催化氧化 $100 \mathrm{ppm}$ 的甲醛. 与 $\mathrm{Pt} / \mathrm{TiO}_{2}$ 相比, 在室温条件下 $\mathrm{Rh} / \mathrm{TiO}_{2}$ 对甲醛的转化 率仅为 $20 \%$ 左右, 而 $\mathrm{Pd} / \mathrm{TiO}_{2}, \mathrm{Au} / \mathrm{TiO}_{2}$ 几乎没有催化 氧化甲醛的能力. 


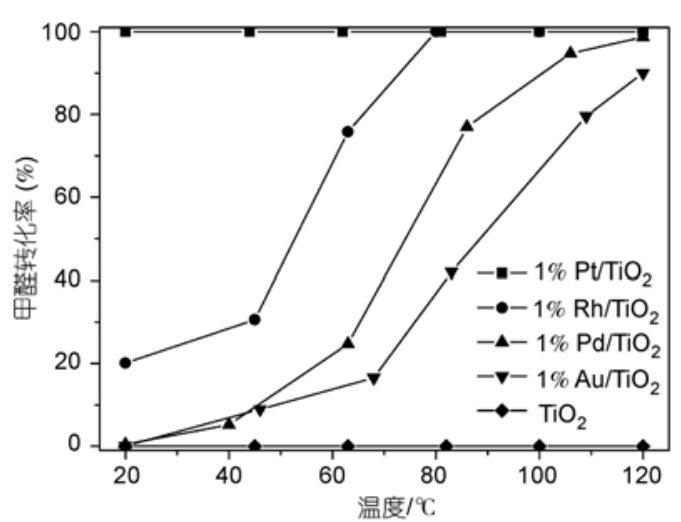

图 $1 \mathrm{TiO}_{2}$ 负载的贵金属催化剂对 HCHO 的 催化氧化活性

在初步研发出具有高活性的 $\mathrm{Pt} / \mathrm{TiO}_{2}$ 催化剂基础 上, 我们同时对其实用性进行了考察. 图 2 给出了室 温条件下, 反应空速以及反应时间对 $\mathrm{Pt} / \mathrm{TiO}_{2}$ 催化氧 化甲醛活性的影响. 可以看出, 随着反应空速由 $50000 \mathrm{~h}^{-1}$ 提高到 $100000 \mathrm{~h}^{-1}$ 以及 $200000 \mathrm{~h}^{-1}$, 达到稳 态时的甲醛转化率逐渐降低，分别为 $100 \%, 97 \%$ 和 $58 \%$. 同时还可以看出，当 $50000 \mathrm{~h}^{-1}$ 空速时，在所考 察的 $24 \mathrm{~h}$ 的反应时间里甲醛的转化率一直维持在 $100 \%$; 但在 100000 和 $200000 \mathrm{~h}^{-1}$ 两种高反应空速条

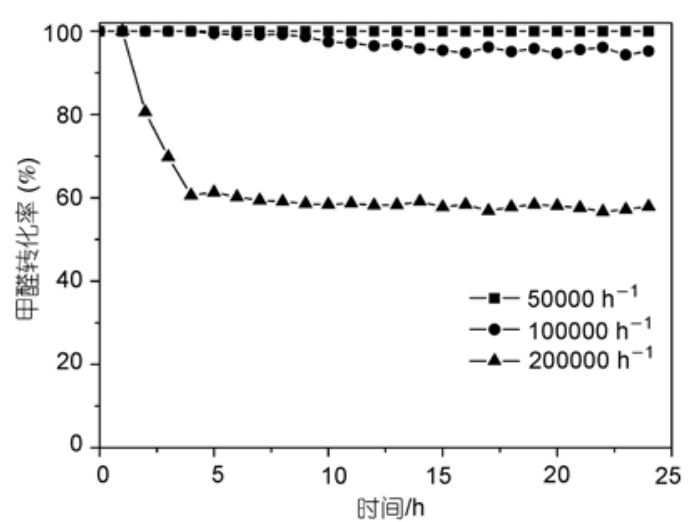

图 2 反应空速以及反应时间对 $1 \% \mathrm{Pt} / \mathrm{TiO}_{2}$ 催化剂催化 氧化 HCHO 活性的影响
件下，反应初始甲醛的转化率也都是 $100 \%$ ，但随着 反应时间的进行, 甲醛转化率会逐渐下降, 最后达到 一个稳定转化率. 值得注意的是, 当甲醛转化率达到 稳态后, 在 3 种空速条件下催化剂都保持了稳定的催 化氧化甲醛活性, 可以说 $\mathrm{Pt} / \mathrm{TiO}_{2}$ 催化剂具有良好的 耐久性.

在上述实验过程中, 当甲醛转化率达到稳态后, 我们对 3 种空速条件下反应前后的碳质量平衡进行 了计算, 结果如表 1 所示. 在计算的过程中, 我们取 了 10 个稳态点, 表中所得数值为 10 次结果的平均值.

根据碳平衡结果, 在 $\mathrm{Pt} / \mathrm{TiO}_{2}$ 催化氧化甲醛过程 中, 室温条件下只发生了 $\mathrm{HCHO}+\mathrm{O}_{2} \rightarrow \mathrm{CO}_{2}+\mathrm{H}_{2} \mathrm{O}$ 的反应, 展现出该催化剂具有极佳的选择性和实用 性能.

\section{$2 \mathrm{TiO}_{2}$ 负载贵金属催化剂的表征}

从活性评价结果可以看出, $\mathrm{TiO}_{2}$ 负载不同的贵金 属的催化剂对甲醛的催化氧化活性具有明显的差异. 为了找到出现差异的原因, 同时揭示 $\mathrm{Pt} / \mathrm{TiO}_{2}$ 催化剂 具有高效催化氧化甲醛的本质原因, 我们还通过 BET 比表面积测量法、X 射线衍射(XRD)、程序升温 还原(TPR)、高分辨透射电子显微谱(HRTEM)等手段 对催化剂进行了一系列表征.

表 2 分别给出了纯 $\mathrm{TiO}_{2}$ 粉末和 $\mathrm{Pt} / \mathrm{TiO}_{2}, \mathrm{Rh} / \mathrm{TiO}_{2}$, $\mathrm{Pd} / \mathrm{TiO}_{2}, \mathrm{Au} / \mathrm{TiO}_{2}$ 催化剂(贵金属负载量为 $1 \%$, 下同) 的比表面积. 由数据可以看出, 纯 $\mathrm{TiO}_{2}$ 粉末的比表面 积约 $49.6 \mathrm{~m}^{2} / \mathrm{g}$, 而负载贵金属之后, 除了 $\mathrm{Au} / \mathrm{TiO}_{2}$ 催 化剂的比表面积下降至 $43.9 \mathrm{~m}^{2} / \mathrm{g}$ 外, 其他催化剂的 比表面并没有发生明显的变化, 只是略有降低. 我们 认为出现比表面积略有下降的现象是由于负载的贵 金属颗粒堵塞载体上的部分微孔所致.

图 3 给出了纯 $\mathrm{TiO}_{2}$ 粉末和 $\mathrm{Pt} / \mathrm{TiO}_{2}, \mathrm{Rh} / \mathrm{TiO}_{2}$, $\mathrm{Pd} / \mathrm{TiO}_{2}, \mathrm{Au} / \mathrm{TiO}_{2}$ 催化剂的 XRD 谱图. 由图可以看出, 除了强度略有差异之外, $\mathrm{TiO}_{2}$ 负载的贵金属催化剂几 乎具有与纯 $\mathrm{TiO}_{2}$ 相同的晶相结构, 谱图中没有发现

表 1 室温下 $1 \% \mathrm{Pt} / \mathrm{TiO}_{2}$ 催化剂催化氧化 $\mathrm{HCHO}$ 过程碳质量平衡

\begin{tabular}{|c|c|c|c|c|c|c|c|c|c|}
\hline \multirow[b]{2}{*}{ 气体 } & \multicolumn{3}{|c|}{$\mathrm{SV}=50000 \mathrm{~h}^{-1}$} & \multicolumn{3}{|c|}{$\mathrm{SV}=100000 \mathrm{~h}^{-1}$} & \multicolumn{3}{|c|}{$\mathrm{SV}=20000 \mathrm{~h}^{-1}$} \\
\hline & $\begin{array}{c}\text { 进口浓度 } \\
(\mathrm{ppm})\end{array}$ & $\begin{array}{c}\text { 出口浓度 } \\
\text { (ppm) }\end{array}$ & 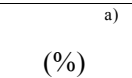 & $\begin{array}{c}\text { 进口浓度 } \\
(\mathrm{ppm})\end{array}$ & $\begin{array}{c}\text { 出口浓度 } \\
(\mathrm{ppm})\end{array}$ & 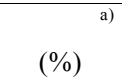 & $\begin{array}{c}\text { 进口浓度 } \\
(\mathrm{ppm})\end{array}$ & $\begin{array}{c}\text { 出口浓度 } \\
\text { (ppm) }\end{array}$ & 选择性 ${ }^{a)}(\%)$ \\
\hline $\mathrm{HCHO}$ & 102 & 0 & - & 101 & 3 & - & 102 & 43 & - \\
\hline $\mathrm{CO}_{2}$ & 5.2 & 107.2 & 100 & 4.9 & 103.6 & 99.7 & 4.8 & 63.7 & 99.8 \\
\hline $\mathrm{CO}$ & 3.2 & 3.2 & - & 2.9 & 2.9 & - & 2.4 & 2.4 & - \\
\hline
\end{tabular}

a) 选择性 $(\%)=\Delta \mathrm{CO}_{2}(\mathrm{ppm}) \times 100 / \Delta \mathrm{HCHO}(\mathrm{ppm}), n=10$ 
表 $2 \mathrm{TiO}_{2}$ 和 $\mathrm{Pt} / \mathrm{TiO}_{2}, \mathrm{Rh} / \mathrm{TiO}_{2}, \mathrm{Pd} / \mathrm{TiO}_{2}, \mathrm{Au} / \mathrm{TiO}_{2}$ 比表面积对比

\begin{tabular}{lc}
\hline 催化剂 & 比表面积 $/ \mathrm{m}^{2} \cdot \mathrm{g}^{-1}$ \\
\hline $\mathrm{TiO}_{2}$ & 49.6 \\
$\mathrm{Pt} / \mathrm{TiO}_{2}$ & 47.4 \\
$\mathrm{Rh} / \mathrm{TiO}_{2}$ & 48.4 \\
$\mathrm{Pd} / \mathrm{TiO}_{2}$ & 46.3 \\
$\mathrm{Au} / \mathrm{TiO}_{2}$ & 43.9 \\
\hline
\end{tabular}

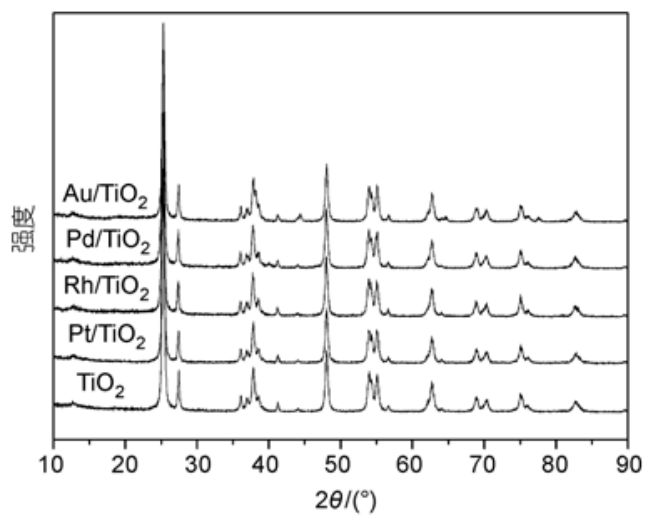

图 $3 \mathrm{TiO}_{2}$ 和 $\mathrm{Pt} / \mathrm{TiO}_{2}, \mathrm{Rh} / \mathrm{TiO}_{2}, \mathrm{Pd} / \mathrm{TiO}_{2}, \mathrm{Au} / \mathrm{TiO}_{2}$ 晶型结构对比

明显的贵金属的晶相峰, 这说明贵金属在载体表面 获得了很好的分散. 图 4 是 $1 \% \mathrm{Pt} / \mathrm{TiO}_{2}$ 超高分辨率电 子显微镜照片, 由图我们可以清晰地分辨出载体 $\mathrm{TiO}_{2}$ 的晶格, 但是也没有发现任何贵金属 $\mathrm{Pt}$ 的微粒. 因为超高分辨率电子显微镜的分辨极限为 $1 \mathrm{~nm}$, 所 以我们认为贵金属 $\mathrm{Pt}$ 在 $\mathrm{TiO}_{2}$ 载体上获得了接近 $100 \%$ 的分散度, 其微粒的粒径已经小于 $1 \mathrm{~nm}$, 这是 $\mathrm{Pt} / \mathrm{TiO}_{2}$ 具有室温催化氧化甲醛活性的重要原因之一.

图 5 是 $\mathrm{TiO}_{2}$ 和 $\mathrm{Pt} / \mathrm{TiO}_{2}$ 的程序升温还原实验. 在 所考察的温度范围内 $\left(25 \sim 500^{\circ} \mathrm{C}\right)$, 纯 $\mathrm{TiO}_{2}$ 载体上没有

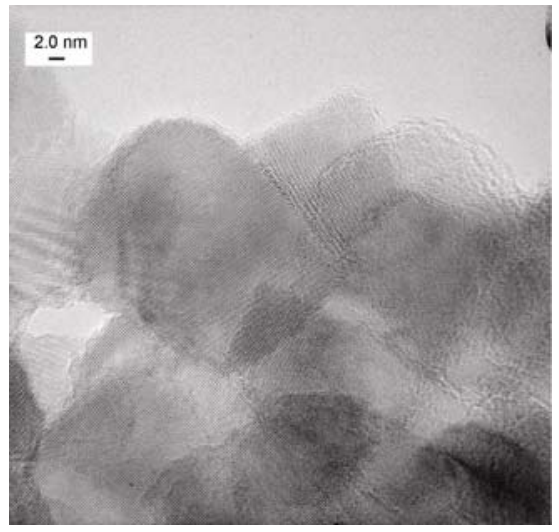

图 $41 \% \mathrm{Pt} / \mathrm{TiO}_{2}$ 催化剂的超高分辨率电子显微镜照片

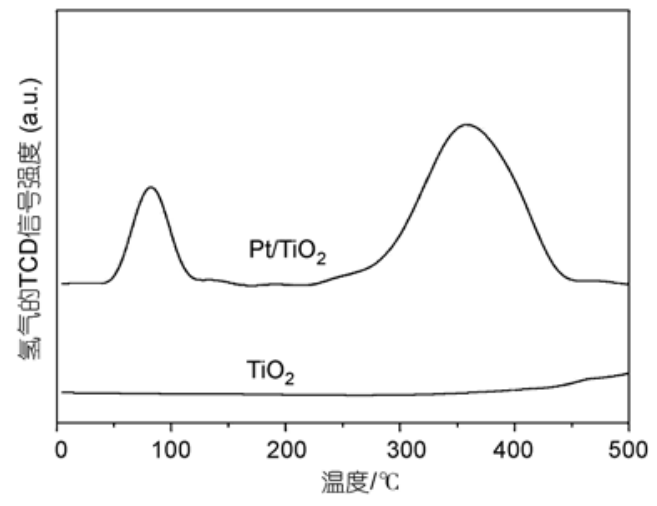

图 $5 \mathrm{TiO}_{2}$ 和 $1 \% \mathrm{Pt} / \mathrm{TiO}_{2}$ 的 TPR 曲线对比

明显的氢气消耗峰. 相比之下, 在 $80^{\circ} \mathrm{C}$ 和 $360^{\circ} \mathrm{C}$ 左右, $\mathrm{Pt} / \mathrm{TiO}_{2}$ 上出现了两个明显的氢气消耗峰. 低温 $80^{\circ} \mathrm{C}$ 时的峰归属于催化剂表面的 $\mathrm{PtO}_{x}$ 相还原为金属态 $\mathrm{Pt}$ 时的氢气消耗; 而 $360^{\circ} \mathrm{C}$ 左右的峰归属于 $\mathrm{TiO}_{2}$ 载体表 面氧还原时的氢气消耗峰, 这说明贵金属 $\mathrm{Pt}$ 的存在 促进了 $\mathrm{TiO}_{2}$ 载体表面氧物种的还原, 这一方面可能 是由于存在氢溢流, 另一方面也不能排除是由于贵 金属-载体相互作用使 $\mathrm{Pt} / \mathrm{TiO}_{2}$ 催化剂表面氧物种的活 性大大增加.

由上述表征结果可以看出，不同贵金属负载的 $\mathrm{TiO}_{2}$ 催化剂之间的比表面积和晶相结构十分相似, 并没有明显的差异, 所以比表面积和晶相结构并不 是造成上述催化剂催化氧化甲醛活性出现巨大差异 的主要原因; 而贵金属 $\mathrm{Pt}$ 在 $\mathrm{TiO}_{2}$ 载体上获得极高的 分散度, 以及贵金属-载体相互作用使表面氧物种的 活性大大增加, 是 $\mathrm{Pt} / \mathrm{TiO}_{2}$ 具有室温活性的两个重要 原因.

\section{$3 \mathrm{TiO}_{2}$ 负载贵金属催化剂室温催化氧化甲 醛机理的研究}

在明确了 $\mathrm{Pt} / \mathrm{TiO}_{2}$ 催化剂具有高效活性的本质原 因的基础上，我们还通过原位红外技术对 $\mathrm{TiO}_{2}$ 负载 的贵金属催化剂室温催化氧化甲醛反应机理进行了 对比研究, 发现由于 4 种催化剂具有不同的活化、分 解甲醛的性能，使甲醛的分解过程停留在不同的反 应阶段, 从而展现出 4 种催化剂之间巨大的活性差 异.

\section{$3.1 \mathrm{Pt} / \mathrm{TiO}_{2}$ 催化剂}

图 6 给出了 $\mathrm{Pt} / \mathrm{TiO}_{2}$ 室温催化氧化甲醛过程中 $\mathrm{Pt} / \mathrm{TiO}_{2}$ 催化剂上随时间变化的红外谱图. 由图 6 可见, 随着导入反应气氛 $\left(\mathrm{O}_{2}+\mathrm{HCHO}+\mathrm{He}\right)$, 在 2062,1657 , 


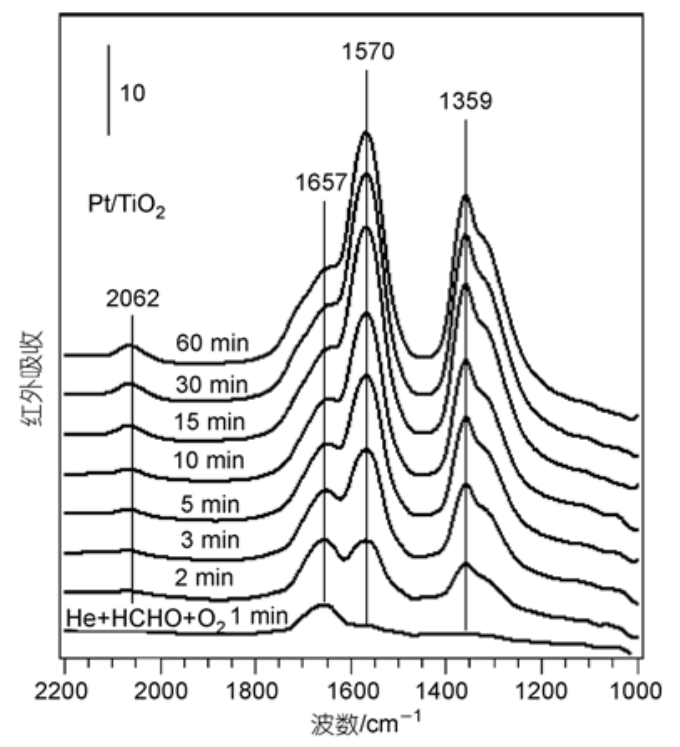

图 6 室温条件下 $\mathrm{Pt} / \mathrm{TiO}_{2}$ 催化剂催化完全氧化 $\mathrm{HCHO}$ 红外谱图

$1570,1359 \mathrm{~cm}^{-1}$ 处出现了红外吸收峰, 峰的强度随着 反应时间的进行逐渐增强，大约 $60 \mathrm{~min}$ 后达到稳态. 在 1359 和 $1570 \mathrm{~cm}^{-1}$ 处的强吸收峰归属于催化剂表面 羧基 $(\mathrm{COO})$ 的对称和非对称伸缩振动，表明在反应 过程中 $\mathrm{Pt} / \mathrm{TiO}_{2}$ 表面形成了高浓度的甲酸盐吸附 物种 ${ }^{[22 ~ 29]}$. 另外, 在 $2062 \mathrm{~cm}^{-1}$ 处还有一个很微弱的 吸收峰, 它归属于在贵金属Pt上线式吸附的CO的振 动 ${ }^{[27 ~ 29]}$. 相比之下, 在相同实验条件下纯 $\mathrm{TiO}_{2}$ 载体上 进行的原位红外实验中, 我们只在 $1657 \mathrm{~cm}^{-1}$ 以及 3000 3500 $\mathrm{cm}^{-1}$ 处观察到因原料气中痕量的水引起 的吸收峰 ${ }^{[30]}$, 因此, $\mathrm{Pt} / \mathrm{TiO}_{2}$ 催化剂上的原位红 外结 果充分说明了 $\mathrm{Pt}$ 对载体 $\mathrm{TiO}_{2}$ 的氧化活性的促进作用.

当上述实验过程(图 6)达到稳态 (60 min)后, 关 闭 $\mathrm{O}_{2}$ 和 $\mathrm{HCHO}$ 气体, 观察单纯在 $\mathrm{He}$ 气吹扫下催化剂 表面吸附物种随时间的变化趋势, 结果见图 7(a). 图 7(b)给出了图 7(a)中甲酸盐物种 $\left(1407 \sim 1240 \mathrm{~cm}^{-1}\right)$ 和 CO物种 (1930 2096 $\left.\mathrm{cm}^{-1}\right)$ 的峰面积随时间的变化趋 势. 由图 7(a)可以看出, 随着氦气的吹扫, 在 1570 , $1359 \mathrm{~cm}^{-1}$ 处甲酸盐物种的红外吸收峰迅速下降, 而 在 2062 和 $1761 \mathrm{~cm}^{-1}$ 处的红外吸收峰却迅速增强, $2062 \mathrm{~cm}^{-1}$ 的峰归属为贵金属 $\mathrm{Pt}$ 上线式吸附的 $\mathrm{CO}$ 物种 的伸缩振动, $1757 \mathrm{~cm}^{-1}$ 处的红外吸收峰归属于贵金 属 $\mathrm{Pt}$ 上桥式吸附的 $\mathrm{CO}$ 物种的伸缩振动 ${ }^{[27]}$. 根据图 7(b), $\mathrm{He}$ 气吹扫 $15 \mathrm{~min}$ 后甲酸盐物种在催化剂表面几
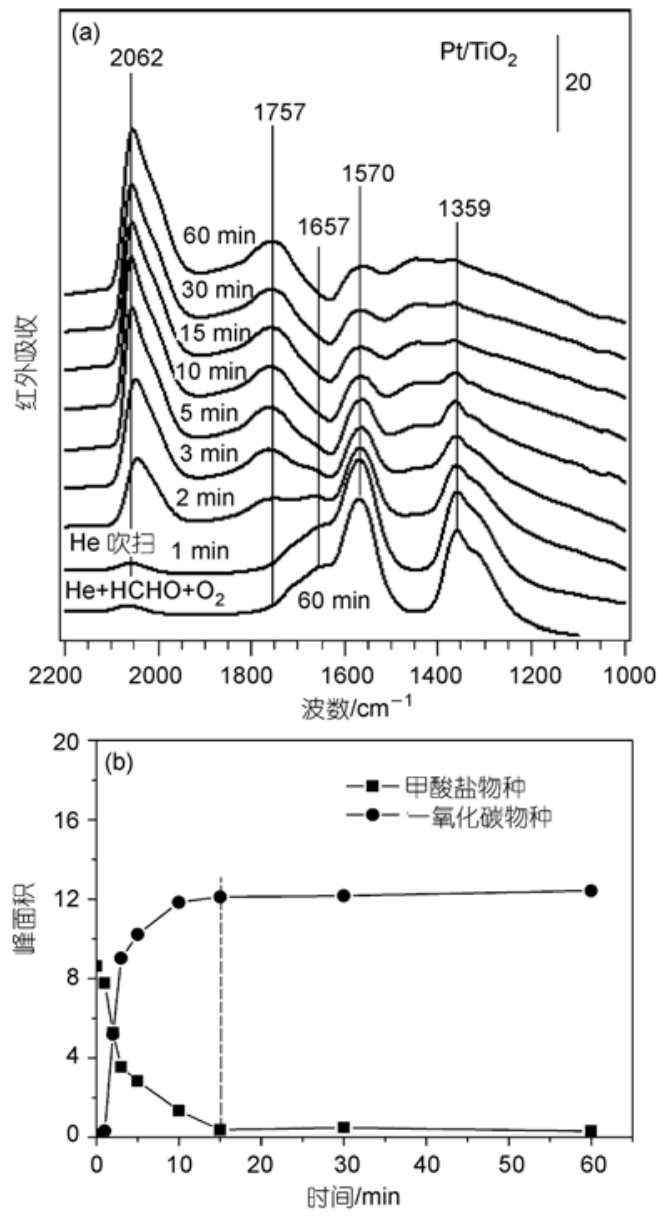

图 7

(a) 反应达到稳态后的 $\mathrm{Pt} / \mathrm{TiO}_{2}$ 在纯氦气吹扫下红外谱图随反应时 间的变化; (b) 图(a)中 1470 1240 $\mathrm{cm}^{-1}$ (甲酸盐物种)和 1930 2096 $\mathrm{cm}^{-1}$ (一氧化碳物种)区间内的积分峰面积随反应时间的变化

乎完全消失, 而 $\mathrm{CO}$ 吸附物种占据了催化剂表面. 根 据以上实验现象, 我们认为甲酸盐吸附物种和 $\mathrm{CO}$ 吸 附物种是室温下 $\mathrm{Pt} / \mathrm{TiO}_{2}$ 催化完全氧化甲醛过程中的 两个重要的中间体, 在氦气的吹扫下 $\mathrm{Pt} / \mathrm{TiO}_{2}$ 表面的 甲酸盐吸附物种会转化为 $\mathrm{CO}$ 吸附物种.

在如图 7 的实验过程达到稳态后, 重新往体系导 入氧气, 考察 $\mathrm{Pt} / \mathrm{TiO}_{2}$ 表面 $\mathrm{CO}$ 吸附物种对 $\mathrm{O}_{2}$ 的反应 活性, 红外谱图如图 8 所示. 可以看出, 随着氧气的 导入, CO 吸附物种在 2062 和 $1757 \mathrm{~cm}^{-1}$ 处的红外吸 收峰迅速下降, 大约 $5 \mathrm{~min}$ 后就在催化剂表面完全消 失. 这说明在 $\mathrm{Pt} / \mathrm{TiO}_{2}$ 上, 室温下 $\mathrm{CO}$ 吸附物种具有与 氧气很强的反应活性. 另外结合图 6 的实验结果可 见，在甲醛氧化过程中, $\mathrm{Pt} / \mathrm{TiO}_{2}$ 表面被大量的甲酸盐 物种所占据, 而只有微量的 $\mathrm{CO}$ 物种存在, 说明在室 


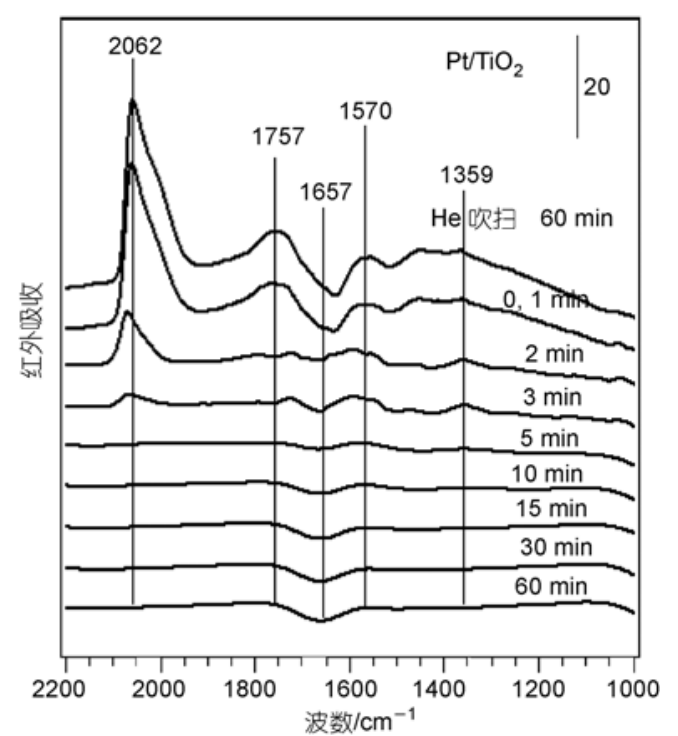

图 $81 \% \mathrm{Pt} / \mathrm{TiO}_{2}$ 催化剂表面 $\mathrm{CO}$ 吸附物种对 $\mathrm{O}_{2}$ 的 反应活性

温下 $\mathrm{Pt} / \mathrm{TiO}_{2}$ 催化氧化甲醛过程中, 甲酸盐物种向 $\mathrm{CO}$ 物种的转化步骤是整个反应的速控步骤.

\section{$3.2 \mathrm{Rh} / \mathrm{TiO}_{2}, \mathrm{Pd} / \mathrm{TiO}_{2}$ 和 $\mathrm{Au} / \mathrm{TiO}_{2}$ 催化剂}

在 $\mathrm{Rh} / \mathrm{TiO}_{2}, \mathrm{Pd} / \mathrm{TiO}_{2}$ 和 $\mathrm{Au} / \mathrm{TiO}_{2}$ 催化剂上同时进 行了上述 3 种实验. 当通入 $\mathrm{O}_{2}+\mathrm{HCHO}+\mathrm{He} 3$ 种气体 $60 \mathrm{~min}$ 后, 4 种催化剂的红外谱图对比如图 9 所示. 可 以看出, 在 $\mathrm{Pt} / \mathrm{TiO}_{2}$ 和 $\mathrm{Rh} / \mathrm{TiO}_{2}$ 上都有占据主导地位的 高浓度甲酸盐物种形成, 只是在 $\mathrm{Rh} / \mathrm{TiO}_{2}$ 上甲酸盐物 种的浓度比在 $\mathrm{Pt} / \mathrm{TiO}_{2}$ 上有所降低. 相比之下, 在

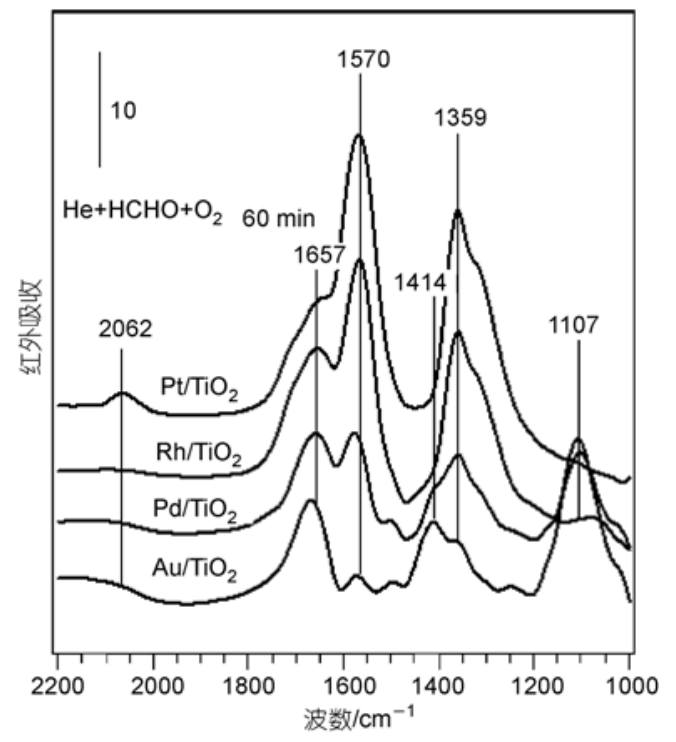

图 9 室温下 $\mathrm{Pt} / \mathrm{TiO}_{2}, \mathrm{Rh} / \mathrm{TiO}_{2}, \mathrm{Pd} / \mathrm{TiO}_{2}$ 和 $\mathrm{Au} / \mathrm{TiO}_{2}$ 催化 完全氧化 HCHO 红外谱图对比
$\mathrm{Pd} / \mathrm{TiO}_{2}$ 和 $\mathrm{Au} / \mathrm{TiO}_{2}$ 催化剂上, 甲酸盐物种的吸收峰强 度明显降低, 已不是占主导地位的表面物种; 而在 1107 和 $1414 \mathrm{~cm}^{-1}$ 出现的二甲酰物种 $\left(\mathrm{H}_{2} \mathrm{CO}_{2}\right)$ 成为 $\mathrm{Pd} / \mathrm{TiO}_{2}$ 和 $\mathrm{Au} / \mathrm{TiO}_{2}$ 催化剂表面的主要吸附物种 26,27$]$. 二甲酰进一步氧化就能在催化剂表面形成甲酸盐物 种, 因此二甲酰物种是甲酸盐物种的前驱体. 根据上 述分析可知, 4 种催化剂催化氧化甲醛形成甲酸盐物 种的活性顺序是: $\mathrm{Pt} / \mathrm{TiO}_{2}>\mathrm{Rh} / \mathrm{TiO}_{2}>\mathrm{Pd} / \mathrm{TiO}_{2}>$ $\mathrm{Au} / \mathrm{TiO}_{2}$, 这与催化剂活性评价结果相符合.

在氦气吹扫 $60 \mathrm{~min}$ 后, $\mathrm{Pt} / \mathrm{TiO}_{2}, \mathrm{Rh} / \mathrm{TiO}_{2}, \mathrm{Pd} / \mathrm{TiO}_{2}$ 和 $\mathrm{Au} / \mathrm{TiO}_{2}$ 的红外谱图对比见图 10. 可以看出, $\mathrm{Pt} / \mathrm{TiO}_{2}$ 表面的甲酸盐吸附物种 $\left(1359,1570 \mathrm{~cm}^{-1}\right)$ 几乎 完全转化为 $\mathrm{CO}$ 吸附物种 $\left(2062 \mathrm{~cm}^{-1}\right)$; 但在 $\mathrm{Rh} / \mathrm{TiO}_{2}$ 催化剂上, 甲酸盐物种依然存在, 同时仅有微量的 $\mathrm{CO}$ 物种生成, 这表明在 $\mathrm{Rh} / \mathrm{TiO}_{2}$ 上的甲酸盐物种难 以分解形成 $\mathrm{CO}$ 吸附物种. 更明显的是, $\mathrm{Pd} / \mathrm{TiO}_{2}$ 和 $\mathrm{Au} / \mathrm{TiO}_{2}$ 上, 除了水的吸收峰的强度 $\left(1657 \mathrm{~cm}^{-1}\right)$ 有所 降低之外, 红外谱图与氦气吹扫前并没有明显的变 化, 说明在 $\mathrm{Pd} / \mathrm{TiO}_{2}$ 和 $\mathrm{Au} / \mathrm{TiO}_{2}$ 上催化氧化甲醛的反 应已经终止. 接下来重新往体系加入氧气, $60 \mathrm{~min}$ 后, 如图 11 所示, $\mathrm{Pt} / \mathrm{TiO}_{2}$ 表面的 $\mathrm{CO}$ 吸附物种完全消失, 而 $\mathrm{Rh} / \mathrm{TiO}_{2}, \mathrm{Pd} / \mathrm{TiO}_{2}$ 和 $\mathrm{Au} / \mathrm{TiO}_{2}$ 的红外谱图与氧气吹 扫前仍然没有明显变化, 说明在这些催化剂上的二 甲酰、甲酸盐和 $\mathrm{CO}$ 吸附物种与 $\mathrm{O}_{2}$ 的反应活性都非 常低.

根据原位红外实验结果, 我们得出了如图 12 所

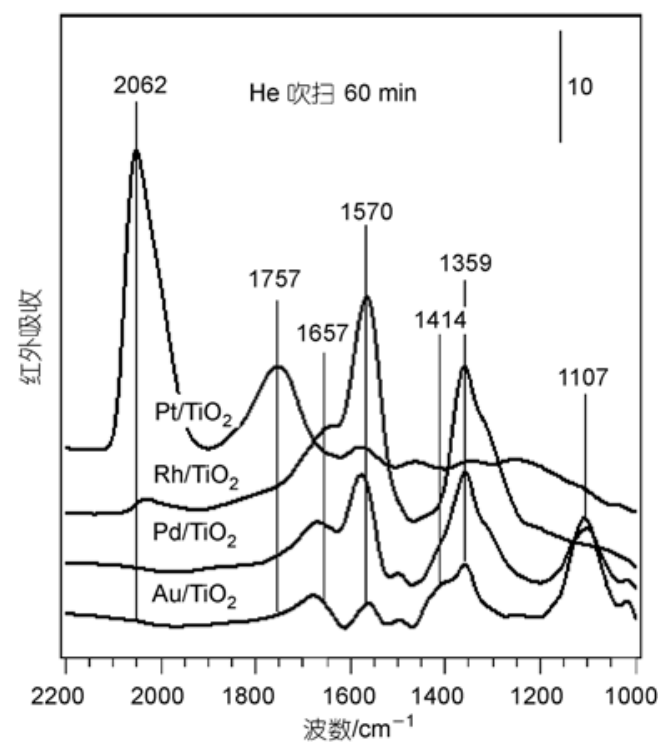

图 10 氦气吹扫下 $\mathrm{Pt} / \mathrm{TiO}_{2}, \mathrm{Rh} / \mathrm{TiO}_{2}, \mathrm{Pd} / \mathrm{TiO}_{2}$ 和 $\mathrm{Au} / \mathrm{TiO}_{2}$ 的红外谱图对比 


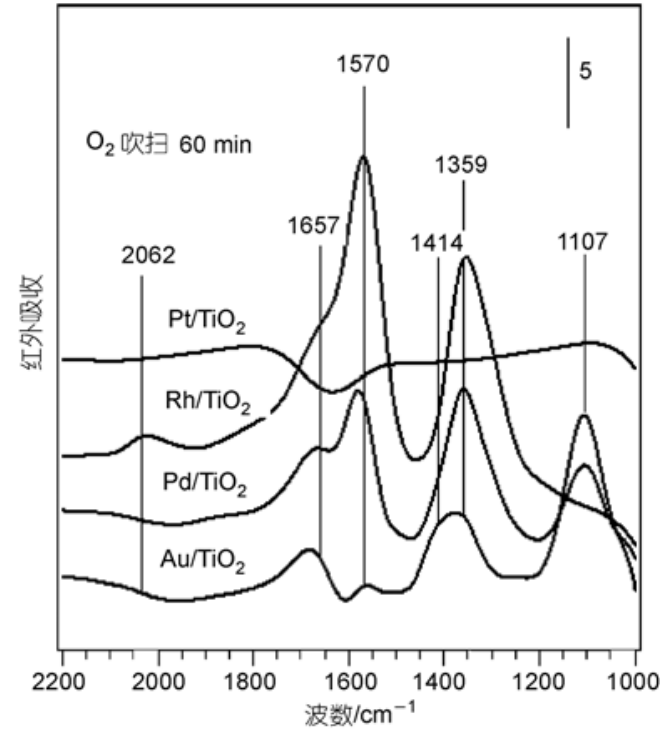

图 11 氧气吹扫下 $\mathrm{Pt} / \mathrm{TiO}_{2}, \mathrm{Rh} / \mathrm{TiO}_{2}, \mathrm{Pd} / \mathrm{TiO}_{2}$ 和 $\mathrm{Au} / \mathrm{TiO}_{2}$ 的红外谱图对比

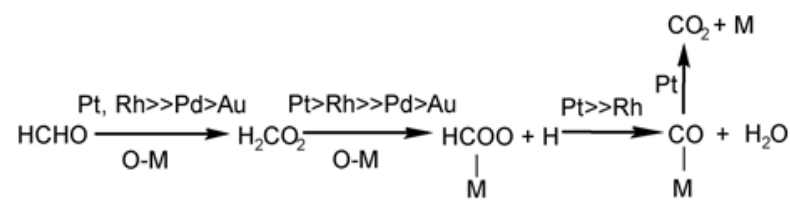

图 12 室温下 $\mathrm{TiO}_{2}$ 负载贵金属催化剂催化完全氧化 HCHO反应机理 ${ }^{[13]}$

示的室温下 $\mathrm{Pt} / \mathrm{TiO}_{2}, \mathrm{Rh} / \mathrm{TiO}_{2}, \mathrm{Pd} / \mathrm{TiO}_{2}$ 和 $\mathrm{Au} / \mathrm{TiO}_{2}$ 催化 氧化甲醛反应机理. 二甲酰、甲酸盐和 $\mathrm{CO}$ 吸附物种 是催化氧化甲醛反应的 3 个重要的反应中间体; 甲醛 首先在催化剂表面被氧化为表面二甲酰物种, 然后 进一步被氧化为甲酸盐物种, 接着甲酸盐物种会转 化为表面 $\mathrm{CO}$ 吸附物种, 随后表面 $\mathrm{CO}$ 吸附物种会迅
速与氧气反应生成最终产物 $\mathrm{CO}_{2}$, 同时空出活性位置. 该机理合理地解释了 4 种催化剂具有不同催化氧化 甲醛活性的原因. 在 $\mathrm{Pt} / \mathrm{TiO}_{2}$ 催化剂上, 甲酸盐物种 很容易形成, 而且甲酸盐可以分解形成 $\mathrm{CO}$ 吸附物种, 然后被氧化生成 $\mathrm{CO}_{2}$, 所以 $\mathrm{Pt} / \mathrm{TiO}_{2}$ 具有室温氧化甲 醛的活性; 在 $\mathrm{Rh} / \mathrm{TiO}_{2}$ 催化剂上, 甲酸盐物种虽然很 容易形成, 但甲酸盐难以分解形成 CO 吸附物种, 因 此 $\mathrm{Rh} / \mathrm{TiO}_{2}$ 的活性远低于 $\mathrm{Pt} / \mathrm{TiO}_{2}$; 而对于 $\mathrm{Pd} / \mathrm{TiO}_{2}$ 和 $\mathrm{Au} / \mathrm{TiO}_{2}$ 来说, 二甲酰容易在催化剂表面形成, 但甲 酸盐物种难以生成, 而且甲酸盐也不能分解为 $\mathrm{CO}$ 吸 附物种, 所以二者的活性又远低于 $\mathrm{Rh} / \mathrm{TiO}_{2}$.

\section{4 实用型 $\mathrm{Pt} / \mathrm{TiO}_{2}$ 催化剂、甲醛净化功能组} 件和空气净化器的开发和应用

首先, 对粉体催化剂的实验室制备工艺进行放 大, 并建立了适应工业生产线的批量生产工艺. 然后, 得到的粉体催化剂经过成型工艺制成实用型催化剂. 在我们组建的工业生产线上, $\mathrm{Pt} / \mathrm{TiO}_{2}$ 粉体催化剂主要 通过原料混合、溶液旋蒸、干燥、焙烧、活化等步骤 制成，再经过制浆工艺涂覆到蜂窝陶瓷等载体上制 成实用型 $\mathrm{Pt} / \mathrm{TiO}_{2}$ 催化剂, 并进一步组装成甲醛净化 功能组件, 最后设计制作成空气净化器和在通风管 道上应用的净化机. 图 13 是我们研制的净化功能组 件和空气净化器. 这种空气净化器已经应用于 2008 年北京奥运会中心区安保指挥中心和公共区管委会, 并在日前上市销售.

图 14 是在 $3 \mathrm{~m}^{3}$ 标准净化测试仓内测试净化器净 化甲醛的效果，同时测试了甲醛和二氧化碳含量的 变化. 试验初始时, 向试验舱内释放甲醛, 甲醛浓度
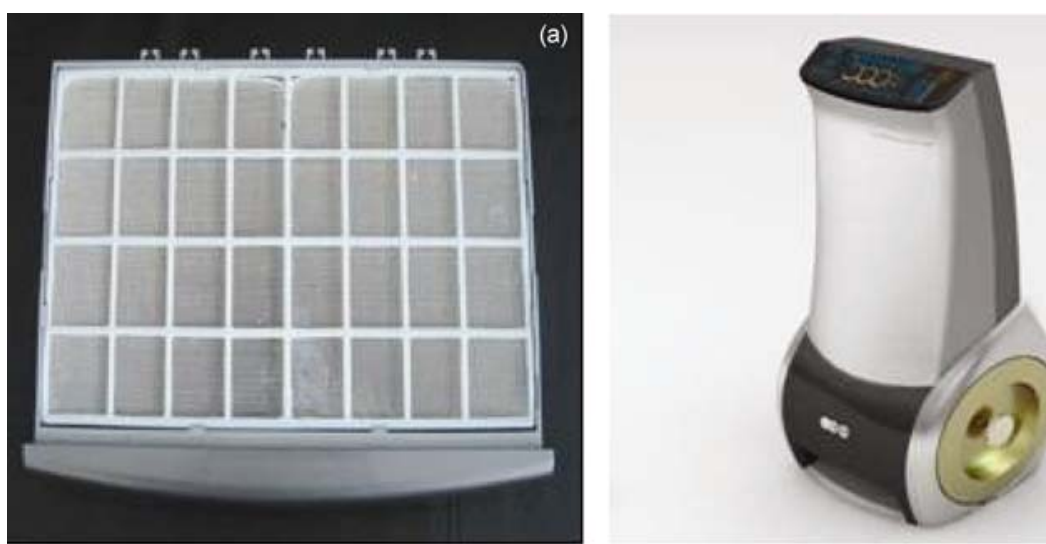

(b)

图 13

(a) 基于 $\mathrm{Pt} / \mathrm{TiO}_{2}$ 催化剂制作的净化功能组件; (b) 基于净化功能组件研制的空气净化器 


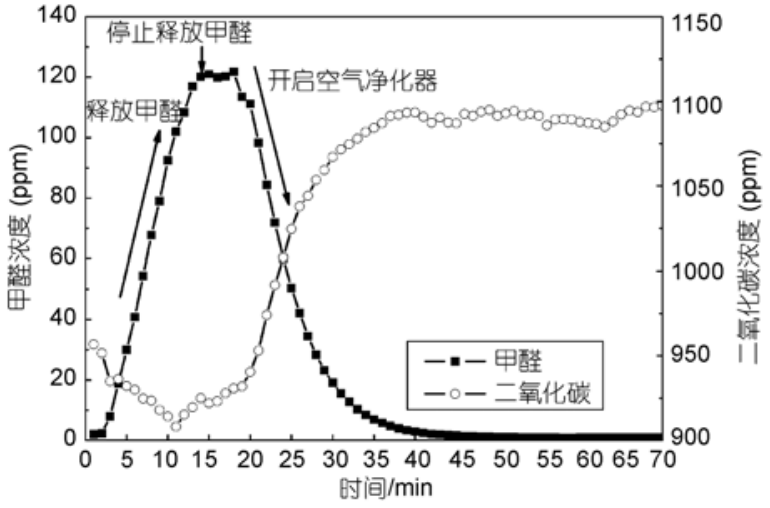

图 $143 \mathrm{~m}^{3}$ 测试舱内空气净化器净化甲醛效果测试 室内空气条件: 温度 $25^{\circ} \mathrm{C}$, 湿度 $60 \%$

快速升高, $15 \mathrm{~min}$ 后, 停止释放甲醛. 待试验仓内甲 醛浓度均匀后, 开启空气净化器, 甲醛浓度快速下降, 同时检测到二氧化碳浓度快速升高, 可以判断甲醛 被氧化成二氧化碳.

\section{5 结束语}

我们通过对 $\mathrm{TiO}_{2}$ 负载的贵金属 $(\mathrm{Au}, \mathrm{Rh}, \mathrm{Pd}, \mathrm{Pt})$
系列催化剂的研究, 在 $\mathrm{Pt} / \mathrm{TiO}_{2}$ 催化剂上实现了室温 催化完全氧化净化甲醛. 一系列的催化剂表征研究 表明，贵金属 $\mathrm{Pt}$ 在 $\mathrm{TiO}_{2}$ 载体上获得了极高的分散度， 同时贵金属-载体相互作用使催化剂表面氧物种的活 性大大增加, 这是 $\mathrm{Pt} / \mathrm{TiO}_{2}$ 催化剂具有室温催化氧化 甲醛活性的两个重要原因. 原位红外实验结果显示, 二甲酰、甲酸盐和 $\mathrm{CO}$ 是 $\mathrm{TiO}_{2}$ 负载的贵金属催化剂 催化氧化甲醛在不同反应阶段的中间产物, 4 种 $\mathrm{TiO}_{2}$ 负载贵金属催化剂由于具有不同的活化、分解甲醛的 性能，使甲醛的分解过程停留在不同的反应阶段，从 而 4 种催化剂呈现出巨大的活性差异. 在实际应用研 究方面, 我们开发了以 $\mathrm{Pt} / \mathrm{TiO}_{2}$ 催化剂为核心的空气 净化器和管道用净化机等产品, 实现了高效、安全和 经济的室内空气甲醛净化, 并应用于北京奥运会中 心区安保指挥中心和公共区管委会的室内空气质量 保障, 给北京奥运会中心区的安保指挥人员提供了 安全舒适的环境. 预计今后这项技术能够进一步惠 及千家万户百姓生活.

\section{参考文献}

1 Yu C, Crump D. A review of the emission of VOCs from polymeric materials used in buildings. Build Environ, 1998, 33(6): 357-374[DOD]

2 Collins J J, Ness R, Tyl R W, et al. A review of adverse pregnancy outcomes and formaldehyde exposure in human and animal studies. Regul Toxicol Pharm, 2001, 34(1): 17-34[DOI]

3 范卫, 王法弟，贾晓东，等。近十年国内有关甲醛的环境与职业危害调查研究. 环境与职业医学, 2004, 21(2): 157一159

4 朱天乐. 室内空气污染控制. 北京: 化学工业出版社, 2002

5 Saleh J M, Hussian S M. Adsorption, desorption and surface decomposition of formaldehyde and acetaldehyde on metal films nickel, palladium and aluminum. J Chem Soc Faraday Trans, 1986, 82: 2221-2234[DOI]

6 Sekine Y, Nishimura A. Removal of formaldehyde from indoor air by passive type air-cleaning materials. Atmos Environ, 2001, 35: 20012007[DOI]

7 Álvarez-Galván M C, de la Peña O’Shea V A, Fierro J L G, et al. Alumina-supported manganese- and manganese-palladium oxide catalysts for VOCs combustion. Catal Commun, 2003, 4: 223-228ㅁD]

8 Tang X F, Li Y G, Huang X M, et al. $\mathrm{MnO}_{x}-\mathrm{CeO}_{2}$ mixed oxide catalysts for complete oxidation of formaldehyde: Effect of preparation method and calcination temperature. Appl Catal B, 2006, 62: 265-273[DOI]

9 Tang X F, Chen J L, Li Y G, et al. Complete oxidation of formaldehyde over Ag/ $\mathrm{MnO}_{x}-\mathrm{CeO}_{2}$ catalysts. Chem Eng J, 2006, 118: 119-125

10 Li C Y, Shen Y N, Jia M L, et al. Catalytic combustion of formaldehyde on gold/iron-oxide catalysts. Catal Commun, 2008, 9: 355-361[DOI]

11 Zhang C B, He H, Tanaka K. Perfect catalytic oxidation of formaldehyde over a $\mathrm{Pt}-\mathrm{TiO}_{2}$ catalyst at room temperature. Catal Commun, 2005, 6: $211-214$ [DOI]

12 Zhang $\mathrm{C} \mathrm{B}, \mathrm{He} \mathrm{H}$, Tanaka K. Catalytic performance and mechanism of a $\mathrm{Pt} / \mathrm{TiO}_{2}$ catalyst for oxidation of formaldehyde at room temperature. Appl Catal B, 2006, 65: 37-43[DOI]

13 Zhang $\mathrm{C} \mathrm{B}, \mathrm{He} \mathrm{H}$. A comparative study of $\mathrm{TiO}_{2}$ supported noble metal catalysts for the oxidation of formaldehyde at room temperature. Catal Today, 2007, 126: 345-350[DOI]

14 He H, Dong X P, Yang M, et al. Catalytic inactivation of SARS coronavirus, Escherichia coli and yeast on solid surface. Catal Commun, 2004, 5(3): 170-172[DOI]

15 问丽珠，陈梅雪，贺泓，等。氧化铝负载银催化剂的杀菌作用. 催化学报, 2005, 26(8): 1122-1126 
16 Boon A Q M, Looij van F, Geus J W. Influence of surface oxygen vacancies on the catalytic activity of copper oxide: Part 1. Oxidation of carbon monoxide. J Mol Catal, 1992, 75(3): 277-291[DOI]

17 Boon A Q M, Looij van F, Geus J W. Influence of surface oxygen vacancies on the catalytic activity of copper oxide Part 2. Oxidation of methane. J Mol Catal, 1992, 75(3): 293-303[DOI]

18 Jernigan G G, Somorjai G A. Carbon monoxide oxidation over three different oxidation states of copper: metallic copper, copper ( I) oxide, and copper (II) oxide — A surface science and kinetic study. J Catal, 1994, 147(2): 567—577[DOI]

19 Rajesh H, Ozkan U S. Complete oxidation of ethanol, acetaldehyde and ethanol/methanol mixtures over copper oxide and copper-chromium oxide catalysts. Ind Eng Chem Res, 1993, 32(8): 1622-1630[DOI]

20 Cordi E M, O’Neill P J, Falconer J L. Transient oxidation of volatile organic compounds on a CuO/ $\mathrm{Al}_{2} \mathrm{O}_{3}$ catalyst. Appl Catal B, 1997, 14(1): $23-36[\mathrm{DOI}]$

21 Zhang C B, Shi X Y, Gao H W, et al. The elimination of formaldehyde over $\mathrm{Cu}-\mathrm{Al}_{2} \mathrm{O}_{3}$ at room temperature. J Environ Sci, 2005, 17(3): $429-432$

22 Boccuzzi F, Chiorino A, Manzoli M. FTIR study of methnol decomposition on gold catalyst for fuel cells. J Power Sources, 2003, 118: $304-310[\mathrm{DOI}]$

23 Millar G J, Rochester C H, Waugh K C. An FTIR study of the adsorption of formic acid and formaldehyde on potassium-promoted $\mathrm{Cu} / \mathrm{SiO}_{2}$ catalysts. J Catal, 1995, 155: 52-58[DOD]

24 Popova G A, Chesalov Y A, Andrushkevich T V, et al. Determination of surface intermediates during the selective oxidation of formaldehyde over V-Ti-O catalyst by in situ FTIR spectroscopy. J Mol Catal, 2000, 158: 345-348[DOI]

25 Liu F Z, Yang M M, Zhou W, et al. In situ transmission difference FTIR spectroscopic investigation on anodic oxidation of methanol in aqueous solution. Electro Chem Comm, 2003, 5: 276-282[DOI]

26 Raskó J, Kecskés T, Kiss J. Formaldehyde formation in the interaction of HCOOH with Pt supported on TiO2. J Catal, 2004, 224: 261$268[\mathrm{DOI}]$

27 Kecskés T, Raskó J, Kiss J. FTIR and mass spectrometric studies on the interaction of formaldehyde with $\mathrm{TiO}_{2}$ supported Pt and Au catalysts. Appl Catal A, 2004, 273: 55-62[DOI]

28 Raskó J. CO-induced surface structural changes of Pt on oxide-supported Pt catalysts studied by DRIFTS. J Catal, 2003, 217: 478-486

29 Liu X S, Korotkikh O, Farauto R. Selective catalytic oxidation of CO in $\mathrm{H}_{2}$ : Structural study of Fe oxide-promoted Pt/alumina catalyst. Appl Catal A, 2002, 226: 293-303

30 张长斌. 氧化催化剂在稀燃尾气 $\mathrm{NO}_{x}$ 选择性催化还原体系和室温催化氧化甲醛中的应用. 博士学位论文. 北京: 中国科学 院研究生院, 2005

\title{
A comparative study of noble metal catalysts for the oxidation of formaldehyde and air purification in indoor environment at room temperature
}

\author{
ZHANG ChangBin ${ }^{1}$, HE Hong ${ }^{1}$, WANG Lian ${ }^{1}$, JIANG Feng $^{2}$, XING Huan ${ }^{2}$, ZHAO Qian $^{2}$ \\ \& $\mathrm{BAO} \mathrm{Wei}^{3}$ \\ ${ }^{1}$ Research Center for Eco-Environmental Sciences, Chinese Academy of Sciences, Beijing 100085, China; \\ ${ }^{2}$ Beijing YA DU Science \& Technology Co., Ltd., Beijing 102206, China; \\ ${ }^{3}$ Beijing Xin Ao Group Co., Ltd., Beijing 100029, China
}

With more and more concern on indoor environmental pollution, many materials and/or techniques with high efficiency, safety and economy are developed. In this study, we introduced the processes of catalytic oxidation of indoor $\mathrm{HCHO}$. The catalytic activities for a complete oxidation of $\mathrm{HCHO}$ over noble metal catalysts with oxygen at room temperature were evaluated. The catalyst of $\mathrm{Pt}_{\mathrm{TiO}} \mathrm{T}_{2}$ showed a high activity for $\mathrm{HCHO}$ oxidation. Based on the experimental results, the mechanism for the catalytic oxidation of $\mathrm{HCHO}$ over noble metal catalysts was proposed. The producing processes of catalyst module for $\mathrm{HCHO}$ purification were exploited and a new type of air purifier was developed, which can be used in room and ventilating duct. This air purifier can effectively and safely remove $\mathrm{HCHO}$ at room temperature without additional running cost. The products were employed in air purification for the 2008 Olympic Games in Beijing. 